\title{
Study of Diet Plans and Different Diseases
}

\section{Murium Sultan', Misbah Zikria1, Muhammad Farooq ${ }^{2 *}$, Iftikhar Ahmed Solangi ${ }^{2}$ and Gulsaya Nuzzhassarova ${ }^{2}$}

${ }^{1}$ Institute of Home and Food Science Government College, University Faisalabad, Pakistan

${ }^{2}$ College of Food Science and Engineering, Northwest A \& F University Yangling, Shaanxi, China

*Corresponding Author: Muhammad Farooq, College of Food Science and Engineering, Northwest A \& F University Yangling, Shaanxi, China.

Received: November 07, 2019; Published: November 18, 2019

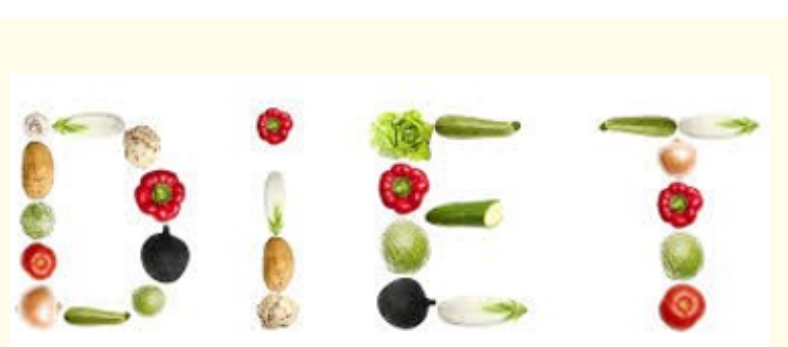

Figure
Preparation of diet plan

The exchange list

\begin{tabular}{|l|c|c|c|c|}
\hline Food Group & $\begin{array}{c}\text { Energy } \\
\text { (Kcals) }\end{array}$ & $\begin{array}{c}\text { Carbohy- } \\
\text { drates (gs) }\end{array}$ & $\begin{array}{c}\text { Protein } \\
\text { (gs) }\end{array}$ & $\begin{array}{c}\text { Fats } \\
\text { (gs) }\end{array}$ \\
\hline Milk & 150 & 12 & 8 & $0-8(4)$ \\
\hline Fruit & 60 & 15 & 0 & 0 \\
\hline Vegetables & 25 & 5 & 2 & 0 \\
\hline Starch & 80 & 15 & 3 & $0-1(1)$ \\
\hline Meat & 75 & 0 & 7 & $0-8(4)$ \\
\hline Fat & 45 & 0 & 0 & 5 \\
\hline
\end{tabular}

Preparation of diet plan by given ADMR

Prepare Diet Plan for given diseases of 2000 Kcals according to following ADMR.
Carbohydrates: $50 \%$

Protein: 20\%

Fats: $30 \%$

Step 1

Calculate the number of grams of Carbohydrates, Protein and Fats. No. of grams of Carbohydrates: $2000 / 100 \times 50=250$ grams No. of grams of Proteins: $2000 / 100 \times 20=100$ grams No. of grams of Fats: $2000 / 100 \times 30=67$ gram Step 2

Calculate the number of servings from each food group.

\begin{tabular}{|l|l|}
\hline Food Group & No of Servings \\
\hline Milk & 2 servings \\
\hline Fruit & 3 servings \\
\hline Vegetables & 4 servings \\
\hline Starch & 11 servings \\
\hline Meat & 6 servings \\
\hline Fat & 5 servings \\
\hline
\end{tabular}

Step 3

Divide the no of servings into meal timings.

\begin{tabular}{|l|c|c|c|c|c|c|}
\hline Exchanges & Breakfast & Snack Time 1 & Lunch & Snack Time 2 & Dinner & Snack Time 3 \\
\hline Starch (11) & 2 & 1 & 3 & 1 & 3 & 1 \\
\hline Vegetable (4) & - & - & 2 & - & 2 & \\
\hline Fruit (3) & E.W & E.W & E.W & E.W & E.W & E.W \\
\hline Milk (2) & 1 & - & - & - & - & 1 \\
\hline Meat (6) & 1 & - & 3 & - & 2 & - \\
\hline Fats (5) & 1 & - & 2 & - & 2 & - \\
\hline
\end{tabular}

Table 3

E.W $=$ Everywhere 
Step 4

Prepare the Diet Plan according to given no of servings in the meal plan.

Diet plan for dysphagia

\begin{tabular}{|l|c|c|c|c|c|}
\hline Day $\mathbf{1}$ and 3 & $\begin{array}{c}\text { Estimated Energy } \\
\text { (Kcals) }\end{array}$ & Day 2,4, 6 & $\begin{array}{c}\text { Estimated Energy } \\
\text { (Kcals) }\end{array}$ & Day 5 and 7 & $\begin{array}{c}\text { Estimated Energy } \\
\text { (Kcals) }\end{array}$ \\
\hline Oatmeal & 140 & Pasta & 240 & 2 toast slice & 160 \\
Soft bread & 160 & custard & 150 & Cheese omelets & 145 \\
Russian salad cream & 260 & & & Pineapple juice & 60 \\
\hline Total & 560 & ------- & 390 & ------- & 365 \\
\hline
\end{tabular}

Breakfast

\begin{tabular}{|l|c|c|c|c|c|}
\hline Day 1 and 3 & $\begin{array}{c}\text { Estimated Energy } \\
\text { (Kcals) }\end{array}$ & Day 2,4 and 6 & $\begin{array}{c}\text { Estimated Energy } \\
\text { (Kcals) }\end{array}$ & Day 5 and 7 & $\begin{array}{c}\text { Estimated Energy } \\
\text { (Kcals) }\end{array}$ \\
\hline 2 slice of toast & 160 & 2 slice soft bread & 160 & Omelet & 75 \\
Omelet & 145 & Omelet & 145 & White bread 2 slices & 180 \\
Apple juice & 60 & Apple juice & 60 & Milk & 150 \\
\hline Total & 365 & --------- & 365 & ------- & 405 \\
\hline
\end{tabular}

Lunch

\begin{tabular}{|c|c|c|c|c|c|}
\hline Day 1 and 3 & $\begin{array}{l}\text { Estimated Energy } \\
\text { (Kcals) }\end{array}$ & Day 2,4 and 6 & $\begin{array}{c}\text { Estimated Energy } \\
\text { (Kcals) }\end{array}$ & Day 5 and 7 & $\begin{array}{c}\text { Estimated Energy } \\
\text { (Kcals) }\end{array}$ \\
\hline Steak fries & & $\begin{array}{c}\text { Soft moist meat+veg } \\
\text { gravy }\end{array}$ & 290 & $\begin{array}{l}\text { Soft cooked } \\
\text { vegetables }\end{array}$ & 140 \\
\hline $\begin{array}{l}\text { Canned chicken } \\
\text { Noodles meat sauce }\end{array}$ & $\begin{array}{l}150 \\
310\end{array}$ & Soft chapatti & 160 & Soft bread & 160 \\
\hline Total & 772 & ----------------- & 450 & ----------------- & 300 \\
\hline
\end{tabular}

Dinner

\begin{tabular}{|c|c|c|c|c|c|c|}
\hline Days & Snack Time 1 & $\begin{array}{c}\text { Estimated } \\
\text { Energy } \\
\text { (Kcals) }\end{array}$ & Snack Time 1 & $\begin{array}{l}\text { Estimated } \\
\text { Energy } \\
\text { (Kcals) }\end{array}$ & Snack Time 1 & $\begin{array}{l}\text { Estimated } \\
\text { Energy } \\
\text { (Kcals) }\end{array}$ \\
\hline Day 1 and 3 & Cold dry cereals & 80 & $\begin{array}{l}\text { Hamburger canned } \\
\text { fruit }\end{array}$ & 60 & Milk with +Ispaghol & 230 \\
\hline Day 2,4 and 6 & Chicken sandwich & 275 & $\begin{array}{c}\text { Orange juice } \\
\text { Mashed potatoes }\end{array}$ & $\begin{array}{l}60 \\
50\end{array}$ & Milk with +Ispaghol & 230 \\
\hline Day 5 and 7 & $\begin{array}{l}\text { Noodles meat } \\
\text { sauce }\end{array}$ & 310 & $\begin{array}{c}\text { Steak fries } \\
\text { Saltin crackers. }\end{array}$ & $\begin{array}{l}130 \\
160\end{array}$ & Milk +Ispaghol & 230 \\
\hline
\end{tabular}


Snack times

Total Estimated Kcals of Day 1 and 3= 2317

Total Estimated Kcals of Day 2, 4 and 6= 1880

Total Estimated Kcals of Day 5 and 7= 1885.

Diet plan for constipation

\begin{tabular}{|c|c|c|c|c|c|c|c|}
\hline Day 1 and 3 & $\begin{array}{c}\text { Estimated } \\
\text { Energy } \\
\text { (Kcals) }\end{array}$ & Day 2and 5 & $\begin{array}{c}\text { Estimated } \\
\text { Energy } \\
\text { (Kcals) }\end{array}$ & Day 4 and 6 & $\begin{array}{c}\text { Estimated } \\
\text { Energy } \\
\text { (Kcals) }\end{array}$ & Day 7 & $\begin{array}{c}\text { Estimated } \\
\text { Energy } \\
\text { (Kcals) }\end{array}$ \\
\hline $\begin{array}{l}2 \text { slice of bread } \\
\text { Omelets } \\
\text { Apple juice }\end{array}$ & $\begin{array}{c}160 \\
145 \\
60\end{array}$ & $\begin{array}{c}\text { Chapatti } \\
2 \text { egg } \\
\text { Yogurt } \\
\text { Apple juice }\end{array}$ & $\begin{array}{l}160 \\
150 \\
150 \\
60\end{array}$ & $\begin{array}{l}\text { Bowl of whole } \\
\text { grain cereals } \\
\text { Yogurt } \\
\text { Apple juice }\end{array}$ & $\begin{array}{l}150 \\
60\end{array}$ & $\begin{array}{c}2 \text { pancakes }(230 \mathrm{kcal}) \\
1 \text { serving strawberries } \\
1 \text { glass carrot juice } \\
\text { Omelet ( } 2 \text { tbsp. Oil) }\end{array}$ & $\begin{array}{r}180 \\
60 \\
110 \\
165\end{array}$ \\
\hline Total & 365 & ----------- & 520 & --------- & 370 & --------- & 515 \\
\hline
\end{tabular}

Breakfast

\begin{tabular}{|c|c|c|c|c|c|c|c|}
\hline Day 1 and 3 & $\begin{array}{c}\text { Estimated } \\
\text { Energy } \\
\text { (Kcals) }\end{array}$ & Day 2 and 5 & $\begin{array}{c}\text { Estimated } \\
\text { Energy } \\
\text { (Kcals) }\end{array}$ & Day 4 and 6 & $\begin{array}{c}\text { Estimated } \\
\text { Energy } \\
\text { (Kcals) }\end{array}$ & Day 7 & $\begin{array}{c}\text { Estimated } \\
\text { Energy } \\
\text { (Kcals) }\end{array}$ \\
\hline Chapatti & 160 & Bread & 160 & Bran rice & 205 & Chicken sandwich & 435 \\
\hline Cabbage meat & 290 & Shami kabab & 150 & Raita & & Salad & 50 \\
\hline $3 / 4$ yogurt & 150 & Salad & 50 & Meat gravy & 290 & Yoghurt & 150 \\
\hline Salad & 50 & & & & & & \\
\hline Total & 650 & 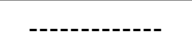 & 360 & - -------- & 495 & |--------- & 635 \\
\hline
\end{tabular}

Lunch

\begin{tabular}{|c|c|c|c|c|c|c|c|}
\hline Day 1 and 3 & $\begin{array}{l}\text { Estimated } \\
\text { Energy } \\
\text { (Kcals) }\end{array}$ & Day 2 and 5 & $\begin{array}{c}\text { Estimated } \\
\text { Energy } \\
\text { (Kcals) }\end{array}$ & Day 4 and 6 & $\begin{array}{c}\text { Estimated } \\
\text { Energy } \\
\text { (Kcals) }\end{array}$ & Day 7 & $\begin{array}{c}\text { Estimated } \\
\text { Energy } \\
\text { (Kcals) }\end{array}$ \\
\hline Chapatti & 160 & Chapatti & 160 & Chapatti & 160 & Brown rice & 180 \\
\hline Chicken gravy & 240 & Potatoes meat & 290 & Potatoes gravy & 220 & $\begin{array}{c}\text { Roasted chicken } \\
\text { Salad }\end{array}$ & $\begin{array}{l}230 \\
50\end{array}$ \\
\hline Total & 400 & - & 450 & ---------- & 380 & - & 460 \\
\hline
\end{tabular}


Dinner

\begin{tabular}{|l|c|c|c|c|c|c|}
\hline Days & Snack Time 1 & $\begin{array}{c}\text { Estimated } \\
\text { Energy } \\
\text { (Kcals) }\end{array}$ & Snack Time 2 & $\begin{array}{c}\text { Estimated Energy } \\
\text { (Kcals) }\end{array}$ & $\begin{array}{c}\text { Estimated } \\
\text { Energy } \\
\text { (Kcals) }\end{array}$ \\
\hline Day 1 and 3 & Apple juice & 120 & Prune juice & 60 & Milk+Ispaghol \\
& Crackers & 160 & 60 & Grape fruit juice & 60 & Milk Biscuit \\
\hline Day 2 and 5 & Prune juice & Sandwich & 230 & 230 \\
\hline Day 4 and 6 & Prune juice & 60 & Crackers & 240 & Milk+Ispaghol \\
\hline Day 7 & Kabab & 200 & 80 & Watermelon & 120 & Milk Biscuit \\
\hline
\end{tabular}

Snack times

Total Estimated Kcals of Day 1 and 3= 1985

Total Estimated Kcals of Day 2 and $5=1875$

Total Estimated Kcals of Day 4 and 6= 1975

Total Estimated Kcals of Day 7= 2040 .

Diet plan for ulcer

\begin{tabular}{|c|c|c|c|c|c|c|c|}
\hline Day 1 and 3 & $\begin{array}{c}\text { Estimated } \\
\text { Energy } \\
\text { (Kcals) }\end{array}$ & Day 2 and 5 & $\begin{array}{c}\text { Estimated } \\
\text { Energy } \\
\text { (Kcals) }\end{array}$ & Day 4 and 6 & $\begin{array}{c}\text { Estimated } \\
\text { Energy } \\
\text { (Kcals) }\end{array}$ & Day 7 & $\begin{array}{c}\text { Estimated } \\
\text { Energy } \\
\text { (Kcals) }\end{array}$ \\
\hline Chapatti & 160 & Bread & 160 & Chicken sandwich & 250 & Bread 3S & 240 \\
\hline Cereals & 250 & 2 egg & 150 & Milk & 150 & Apple & 60 \\
\hline yogurt & 150 & Yogurt & 150 & Melon juice & 60 & Milk & 150 \\
\hline Total & 560 & ------------ & 460 & ----------- & 460 & ------------ & 450 \\
\hline
\end{tabular}

Breakfast

\begin{tabular}{|c|c|c|c|c|c|c|c|}
\hline Day 1 and 3 & $\begin{array}{c}\text { Estimated } \\
\text { Energy } \\
\text { (Kcals) }\end{array}$ & Day 2 and 5 & $\begin{array}{l}\text { Estimated } \\
\text { Energy } \\
\text { (Kcals) }\end{array}$ & Day 4 and 6 & $\begin{array}{l}\text { Estimated } \\
\text { Energy } \\
\text { (Kcals) }\end{array}$ & Day 7 & $\begin{array}{c}\text { Estimated } \\
\text { Energy } \\
\text { (Kcals) }\end{array}$ \\
\hline $\begin{array}{l}\text { Cooked rice } \\
\text { Vegetables } \\
\text { Gravy } \\
\text { Salad }\end{array}$ & $\begin{array}{c}250 \\
90 \\
90 \\
50\end{array}$ & $\begin{array}{c}\text { Rice } 2 \text { cup } \\
\text { (Cooked with } 1 \text { tbs of oil) } \\
\text { Pulses } \\
\text { salad }\end{array}$ & $\begin{array}{c}150 \\
50\end{array}$ & $\begin{array}{l}\text { Vege mix with } \\
\text { egg } \\
\text { Rotti } 1 \frac{1}{2} \\
\text { Fish }\end{array}$ & $\begin{array}{l}240 \\
150\end{array}$ & $\begin{array}{c}\text { Rotti } 2 \mathrm{~S} \\
\text { Well cook vege } 1 \\
1 / 2 \text { (cook with } 2 \text { tbs } \\
\text { of oil) } \\
\text { Raita } 1 / 2\end{array}$ & 165 \\
\hline Total & 390 & ------------ & 450 & --------. & 590 & -------- & 560 \\
\hline
\end{tabular}


Lunch

\begin{tabular}{|c|c|c|c|c|c|c|c|}
\hline Day 1 and 3 & $\begin{array}{c}\text { Estimated } \\
\text { Energy } \\
\text { (Kcals) }\end{array}$ & Day 2 and 5 & $\begin{array}{c}\text { Estimated } \\
\text { Energy } \\
\text { (Kcals) }\end{array}$ & Day 4 and 6 & $\begin{array}{c}\text { Estimated } \\
\text { Energy } \\
\text { (Kcals) }\end{array}$ & Day 7 & $\begin{array}{c}\text { Estimated } \\
\text { Energy } \\
\text { (Kcals) }\end{array}$ \\
\hline \multirow{5}{*}{$\begin{array}{l}\text { Chicken } \\
\text { sandwich } \\
\text { Ketchup }\end{array}$} & \multirow[t]{2}{*}{275} & \multirow{5}{*}{$\begin{array}{c}\text { Chapatti } \\
\text { Chicken gravy } \\
\text { Custard }\end{array}$} & \multirow{5}{*}{$\begin{array}{l}160 \\
140 \\
150\end{array}$} & \multirow{5}{*}{$\begin{array}{c}\text { Steamed vege } 1 \text { 1/2 } \\
\text { Chapatti } 2 \mathrm{~S} \\
\text { Fresh juice }\end{array}$} & \multirow{5}{*}{$\begin{array}{c}75 \\
320 \\
60\end{array}$} & \multirow{5}{*}{$\begin{array}{c}\text { 2S of rice } \\
\text { (cook with } 1 \text { tbs of oil) } \\
\text { Bowl of daal } \\
\text { (cooked with } 2 \text { tbs of oil) } \\
\text { Fruit juice }\end{array}$} & 160 \\
\hline & & & & & & & 45 \\
\hline & 140 & & & & & & 120 \\
\hline & & & & & & & 90 \\
\hline & & & & & & & 60 \\
\hline Total & 415 & ----------- & 450 & ----------- & 455 & - & 475 \\
\hline
\end{tabular}

Dinner

\begin{tabular}{|l|c|c|c|c|c|c|}
\hline Days & Snack Time 1 & $\begin{array}{c}\text { Estimated } \\
\text { Energy } \\
\text { (Kcals) }\end{array}$ & Snack Time 2 & $\begin{array}{c}\text { Estimated } \\
\text { Energy } \\
\text { (Kcals) }\end{array}$ & $\begin{array}{c}\text { Snack Time 3 } \\
\text { Energy } \\
\text { (Kcals) }\end{array}$ \\
\hline Day 1 and 3 & $\begin{array}{c}\text { Sweet potatoes } \\
\text { Dates }\end{array}$ & $\begin{array}{c}90 \\
6=150\end{array}$ & Pineapple juice & 60 & Milk & 150 \\
\hline Day 2 and 5 & Fresh juice & 160 & $\begin{array}{c}\text { Chicken } \\
\text { Sandwich+pear }\end{array}$ & 275 & 60 & 150 \\
\hline Day 4 and 6 & Mousse & 130 & Medium banana & 60 & Bowl of custard & 200 \\
\hline Day 7 & Fruit chaat & 120 & Mousse & 130 & Milk + Mango & $150+60=210$ \\
\hline
\end{tabular}

\section{Snack times}

Total Estimated Kcals of Day 1 and 3= 1725

Total Estimated Kcals of Day 2 and $5=1905$

Total Estimated Kcals of Day 4 and 6= 1895

Total Estimated Kcals of Day 7= 1945.

Diet plan for gastritis

\begin{tabular}{|c|c|c|c|c|c|c|c|}
\hline Day 1 and 3 & $\begin{array}{c}\text { Estimated } \\
\text { Energy } \\
\text { (Kcals) }\end{array}$ & Day 2 and 5 & $\begin{array}{c}\text { Estimated } \\
\text { Energy } \\
\text { (Kcals) }\end{array}$ & Day 4 and 6 & $\begin{array}{c}\text { Estimated } \\
\text { Energy } \\
\text { (Kcals) }\end{array}$ & Day 7 & $\begin{array}{c}\text { Estimated } \\
\text { Energy } \\
\text { (Kcals) }\end{array}$ \\
\hline $\begin{array}{l}2 \text { slice of bread } \\
\text { Omelets } \\
\text { Yogurt }\end{array}$ & $\begin{array}{l}160 \\
145 \\
150\end{array}$ & $\begin{array}{c}2 \text { bowl of cereal grain } \\
1 \text { cup low fat milk } \\
\text { Egg boiled } \\
1 \text { tbs of mayonnaise }\end{array}$ & $\begin{array}{l}180 \\
150 \\
70 \\
90\end{array}$ & $\begin{array}{l}\text { Tendered chicken } \\
\text { sandwich }\end{array}$ & $\begin{array}{l}150 \\
60\end{array}$ & $\begin{array}{c}\text { Bread 2S } \\
\text { Omelets } \\
\text { (1 tbs of oil) } \\
1 \text { Cup of fruit } \\
\text { with added } \\
\text { Mayonnaise }\end{array}$ & $\begin{array}{l}60 \\
80\end{array}$ \\
\hline Total & 455 & ------------- & 490 & ---------- & 540 & ----------- & 420 \\
\hline
\end{tabular}


Breakfast

\begin{tabular}{|c|c|c|c|c|c|c|c|}
\hline Day 1 and 3 & $\begin{array}{c}\text { Estimated } \\
\text { Energy } \\
\text { (Kcals) }\end{array}$ & Day 2 and 5 & $\begin{array}{c}\text { Estimated } \\
\text { Energy } \\
\text { (Kcals) }\end{array}$ & Day 4 and 6 & $\begin{array}{l}\text { Estimated } \\
\text { Energy } \\
\text { (Kcals) }\end{array}$ & Day 7 & $\begin{array}{l}\text { Estimated } \\
\text { Energy } \\
\text { (Kcals) }\end{array}$ \\
\hline Shami kabab & 275 & $\begin{array}{c}\text { Steamed chicken } 3 \mathrm{~S} \\
\text { Yoghurt } 1 / 2 \mathrm{~S}\end{array}$ & $\begin{array}{l}230 \\
75\end{array}$ & $\begin{array}{l}\text { Lean meat } 2 \mathrm{~S} \\
1 \text { cup fruit juice } \\
\text { Tender vege } 1 \text { cup }\end{array}$ & $\begin{array}{l}150 \\
60 \\
50 \\
\end{array}$ & $\begin{array}{l}\text { Cooked with } \\
2 \text { tbs Oil } \\
\text { Roti } 11 / 2\end{array}$ & 90 \\
\hline Total & 370 & ----------- & 485 & -----.---- & 420 & 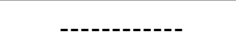 & 560 \\
\hline
\end{tabular}

Lunch

\begin{tabular}{|c|c|c|c|c|c|c|c|}
\hline Day 1 and 3 & $\begin{array}{c}\text { Estimated } \\
\text { Energy } \\
\text { (Kcals) }\end{array}$ & Day 2 and 5 & $\begin{array}{c}\text { Estimated } \\
\text { Energy } \\
\text { (Kcals) }\end{array}$ & Day 4 and 6 & $\begin{array}{c}\text { Estimated } \\
\text { Energy } \\
\text { (Kcals) }\end{array}$ & Day 7 & $\begin{array}{c}\text { Estimated } \\
\text { Energy } \\
\text { (Kcals) }\end{array}$ \\
\hline Pasta & 305 & Soft bread 2S & 160 & Hamburger & 180 & Roti $3 \mathrm{~S}$ whole grain & 240 \\
\hline Apple juice & 60 & $\begin{array}{l}\text { Chicken gravy } \\
\text { Applesauce } 1 \\
\text { cup }\end{array}$ & $\begin{array}{l}230 \\
60\end{array}$ & $\begin{array}{c}\text { Meat salad } \\
\text { Baked potatoes } \\
\text { 1/2 cup }\end{array}$ & $\begin{array}{l}230 \\
160\end{array}$ & $\begin{array}{c}1 \text { cup well-cooked vege } \\
\text { (1 Tbsp. oil) } \\
\text { Grilled meat } 3 \mathrm{~S}\end{array}$ & $\begin{array}{r}95 \\
230\end{array}$ \\
\hline Total & 455 & 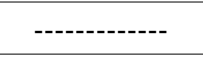 & 450 & ---------- & 570 & - & 565 \\
\hline
\end{tabular}

Dinner

\begin{tabular}{|l|c|c|c|c|c|c|}
\hline Days & Snack Time 1 & $\begin{array}{c}\text { Estimated } \\
\text { Energy } \\
\text { (Kcals) }\end{array}$ & Snack Time 2 & $\begin{array}{c}\text { Estimated } \\
\text { Energy } \\
\text { (Kcals) }\end{array}$ & $\begin{array}{c}\text { Estimated } \\
\text { Energy } \\
\text { (Kcals) }\end{array}$ \\
\hline Day 1 and 3 & $\begin{array}{c}\text { Crackers } \\
\text { banana }\end{array}$ & $60=220$ & Canned fruit & $60=370$ & 150 \\
\hline Day 2 and 5 & Cutted melon 1 cup & 60 & $\begin{array}{c}\text { Strawberry flavored } \\
\text { yoghurt }\end{array}$ & 210 & Milk + biscuit 1S \\
\hline Day 4 and 6 & Mousse & 130 & Tea with biscuits & 160 & 1 cup yoghurt \\
\hline Day 7 & Peach med & 240 & Apple & 60 & Fat free milk \\
\hline
\end{tabular}


Snack times

Total Estimated Kcals of Day 1 and 3= 1995

Total Estimated Kcals of Day 2 and $5=1925$

Total Estimated Kcals of Day 4 and 6= 1970

Total Estimated Kcals of Day 7=1995.

Diet plan for diarrhea

\begin{tabular}{|c|c|c|c|c|c|c|c|}
\hline Day 1 and 3 & $\begin{array}{c}\text { Estimated } \\
\text { Energy } \\
\text { (Kcals) }\end{array}$ & Day 2 and 5 & $\begin{array}{c}\text { Estimated } \\
\text { Energy } \\
\text { (Kcals) }\end{array}$ & Day 4 and 6 & $\begin{array}{c}\text { Estimated } \\
\text { Energy } \\
\text { (Kcals) }\end{array}$ & Day 7 & $\begin{array}{c}\text { Estimated } \\
\text { Energy } \\
\text { (Kcals) }\end{array}$ \\
\hline 1 cup cereal & 80 & Scrambled egg & 75 & Banana shake & 210 & Banana & 60 \\
\hline 2 slice (white) & 160 & Toast & 160 & 2 slice of bread & 160 & One glass milk & 150 \\
\hline Milk & 150 & Milk & 150 & $\begin{array}{c}\text { Egg omelate } \\
\text { (1 tbs oil) }\end{array}$ & 120 & & 160 \\
\hline Egg boiled & 75 & Med. Size apple & 60 & & & 1 cup cereals & 80 \\
\hline Total & 465 & 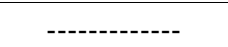 & 445 & - ------- & 490 & - & 450 \\
\hline
\end{tabular}

Breakfast

\begin{tabular}{|c|c|c|c|c|c|c|c|}
\hline Day 1 and 3 & $\begin{array}{c}\text { Estimated } \\
\text { Energy } \\
\text { (Kcals) }\end{array}$ & Day 2 and 5 & $\begin{array}{c}\text { Estimated } \\
\text { Energy } \\
\text { (Kcals) }\end{array}$ & Day 4 and 6 & $\begin{array}{c}\text { Estimated } \\
\text { Energy } \\
\text { (Kcals) }\end{array}$ & Day 7 & $\begin{array}{c}\text { Estimated } \\
\text { Energy } \\
\text { (Kcals) }\end{array}$ \\
\hline Rice 2S & 160 & 2S khichdi & 170 & Roti $1 / 2$ & 240 & Rice 2S & 160 \\
\hline Daal (1 Plate) & 120 & Yoghourt & 150 & $\begin{array}{c}\text { Chicken gravy } \\
\text { (2 tbs oil) }\end{array}$ & 320 & Steamed chicken & 230 \\
\hline Baked potato $1 / 2$ cup & 50 & Salad & 25 & & & Raita 1S & 150 \\
\hline Chicken steamed $2 \mathrm{~S}$ & 150 & Mayonnaise 1tbsp. & 90 & $\begin{array}{c}\text { Salad cooked } \\
\text { tender }\end{array}$ & 50 & Salad & 25 \\
\hline Total & 480 & 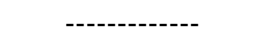 & 435 & --------- & 610 & ----------- & 565 \\
\hline
\end{tabular}

Lunch

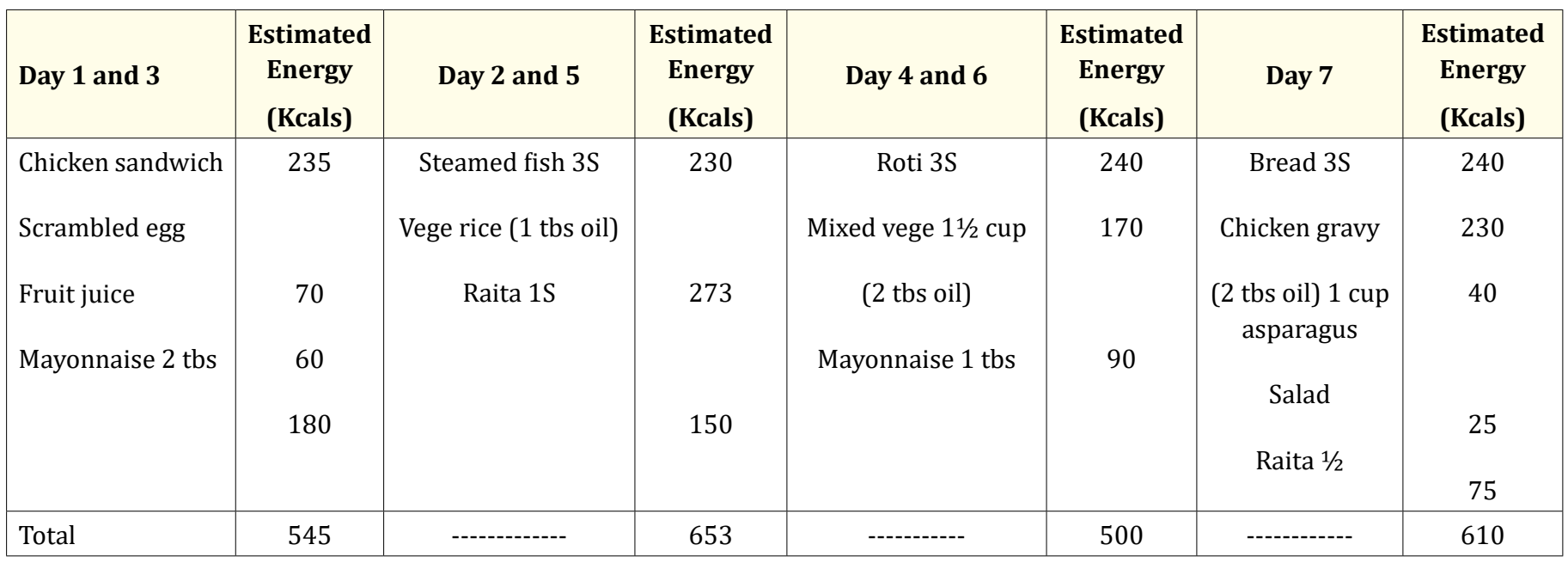

Citation: Murium Sultan., et al. "Study of Diet Plans and Different Diseases". Acta Scientific Nutritional Health 3.12 (2019): 176-190. 
Dinner

\begin{tabular}{|l|c|c|c|c|c|c|}
\hline Days & Snack Time 1 & $\begin{array}{c}\text { Estimated Energy } \\
\text { (Kcals) }\end{array}$ & Snack Time 2 & $\begin{array}{c}\text { Estimated Energy } \\
\text { (Kcals) }\end{array}$ & $\begin{array}{c}\text { Snack Time 3 } \\
\text { Estimated Energy } \\
\text { (Kcals) }\end{array}$ \\
\hline Day 1 and 3 & Flavored Yoghourt & 150 & Banana 2S & 120 & Custard & 200 \\
\hline Day 2 and 5 & 1 banana & 60 & Mousse & 130 & Milk + biscuit & 230 \\
\hline Day 4 and 6 & Orange juice & 60 & Yoghourt & 150 & 1 glass milk & 150 \\
\hline Day 7 & Mousse & 130 & Milk & 150 & Banana 2S \\
\hline
\end{tabular}

Snack times

Total Estimated Kcals of Day 1 and 3= 1960

Total Estimated Kcals of Day 2and 5= 1953

Total Estimated Kcals of Day 4 and 6= 1960

Total Estimated Kcals of Day $7=2025$.

Diet Plan for diabetes

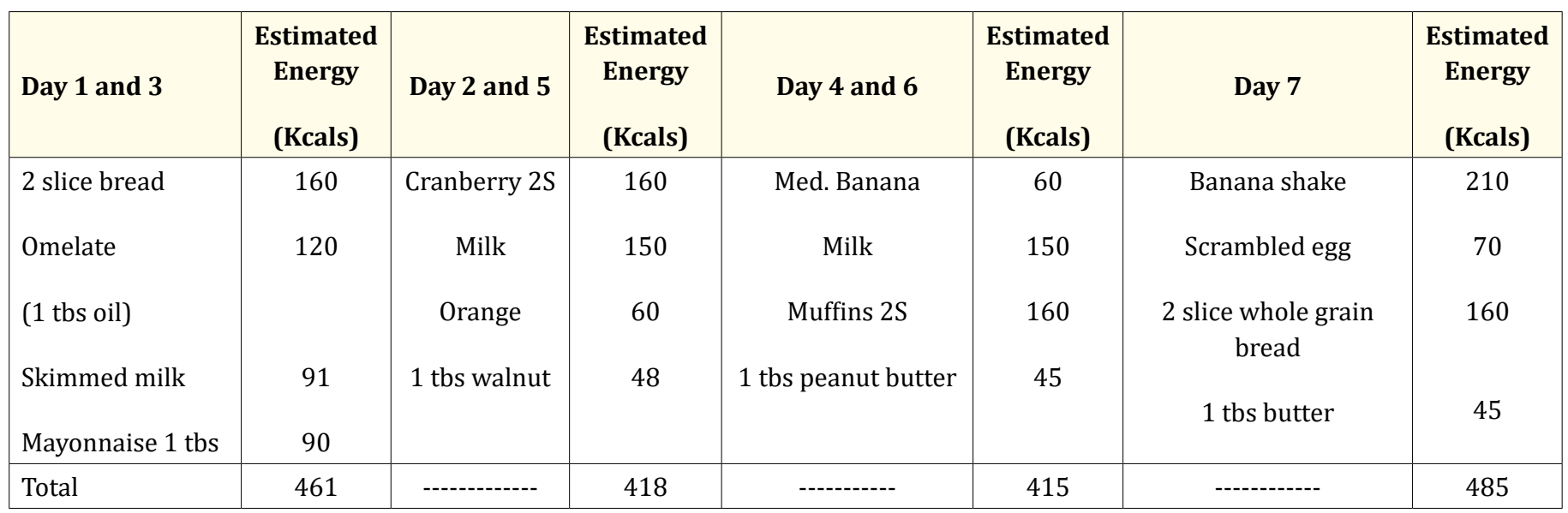

Breakfast

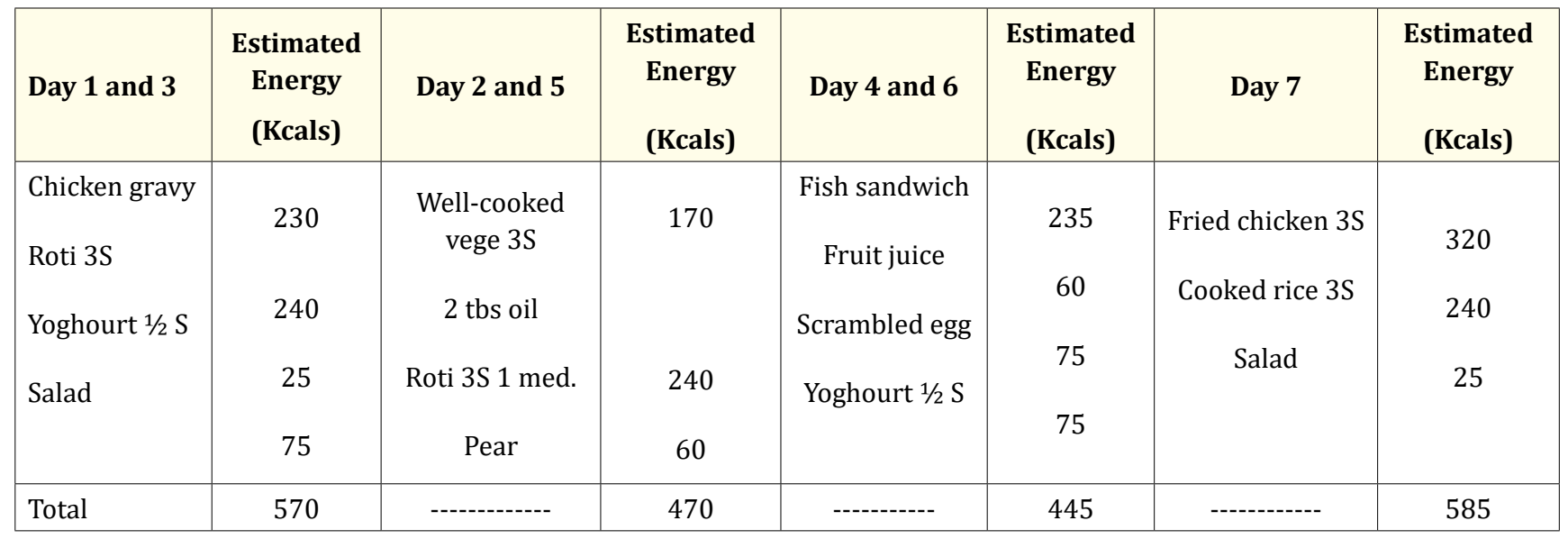


Lunch

\begin{tabular}{|c|c|c|c|c|c|c|c|}
\hline Day 1 and 3 & $\begin{array}{l}\text { Estimated } \\
\text { Energy } \\
\text { (Kcals) }\end{array}$ & Day 2 and 5 & $\begin{array}{l}\text { Estimated } \\
\text { Energy } \\
\text { (Kcals) }\end{array}$ & Day 4 and 6 & $\begin{array}{c}\text { Estimated } \\
\text { Energy } \\
\text { (Kcals) }\end{array}$ & Day 7 & $\begin{array}{c}\text { Estimated } \\
\text { Energy } \\
\text { (Kcals) }\end{array}$ \\
\hline Chicken pasta $2 \mathrm{~S}$ & & Brown rice $2 \mathrm{~S}$ & & 1 cup couscous & & $\begin{array}{l}1 \text { dish chicken and } \\
\text { rice }\end{array}$ & 340 \\
\hline $\begin{array}{l}1 / 2 \text { cup yoghourt } \\
\text { Salad }\end{array}$ & $\begin{array}{l}75 \\
25\end{array}$ & $\begin{array}{l}\text { Steam fish } 3 S \\
\text { Yoghourt } 1 / 2 \mathrm{~S}\end{array}$ & $\begin{array}{l}230 \\
75\end{array}$ & $\begin{array}{l}1 \text { cup cooked } \\
\text { broccoli }\end{array}$ & 50 & $\begin{array}{l}1 \text { cup toasted } \\
\text { cereals }\end{array}$ & 50 \\
\hline & & & & $\begin{array}{l}\text { Lean broiled } \\
\text { steak } \\
\text { Salad } \\
1 / 2 \text { cup yoghurt }\end{array}$ & $\begin{array}{r}230 \\
25 \\
60 \\
\end{array}$ & $\begin{array}{c}2 \text { tbs low fat salad } \\
\text { dressing } \\
\text { Whole bread roll }\end{array}$ & 70 \\
\hline Total & 500 & ------------- & 490 & ----------- & 415 & ------------ & 540 \\
\hline
\end{tabular}

Dinner

\begin{tabular}{|c|c|c|c|c|c|c|}
\hline Days & Snack Time 1 & $\begin{array}{l}\text { Estimated Energy } \\
\text { (Kcals) }\end{array}$ & Snack Time 2 & $\begin{array}{l}\text { Estimated Energy } \\
\text { (Kcals) }\end{array}$ & Snack Time 3 & $\begin{array}{c}\text { Estimated Energy } \\
\text { (Kcals) }\end{array}$ \\
\hline Day 1 and 3 & Almond 1S & 164 & 2 plum & 60 & Milk & 150 \\
\hline Day 2 and 5 & Yoghurt & 150 & Pistachio nut 1S & 158 & Fresh fruit & 60 \\
\hline Day 4 and 6 & $\begin{array}{c}1 \text { whole wheat } \\
\text { pretzel }\end{array}$ & 103 & $\begin{array}{c}\text { blackberries } \\
1 \text { cup }\end{array}$ & 73 & Cheddar cheese & 140 \\
\hline Day 7 & Yoghurt & 150 & $\begin{array}{l}\text { Baked tortilla } \\
\text { chip }\end{array}$ & 180 & Milk & 150 \\
\hline
\end{tabular}

\section{Snack times}

Total Estimated Kcals of Day 1 and 3= 1934

Total Estimated Kcals of Day 2and 5= 1740

Total Estimated Kcals of Day 4 and 6= 1591

Total Estimated Kcals of Day $7=2090$.

Diet plan for hepatitis

\begin{tabular}{|c|c|c|c|c|c|c|c|}
\hline Day 1 and 3 & $\begin{array}{c}\text { Estimated } \\
\text { Energy } \\
\text { (Kcals) }\end{array}$ & Day 2 and 5 & $\begin{array}{c}\text { Estimated } \\
\text { Energy } \\
\text { (Kcals) }\end{array}$ & Day 4 and 6 & $\begin{array}{c}\text { Estimated } \\
\text { Energy } \\
\text { (Kcals) }\end{array}$ & Day 7 & $\begin{array}{c}\text { Estimated } \\
\text { Energy } \\
\text { (Kcals) }\end{array}$ \\
\hline Bread 2 slices & 160 & Chicken sandwich & 320 & Toasted bread & 225 & Egg & 70 \\
\hline Milk fat free & 150 & Apple juice & & Peach juice & 60 & Bread 2 slices & 160 \\
\hline Egg & 70 & Bowl of cereal & 60 & Milk $1 / 2$ cup & 75 & Milk & 150 \\
\hline Bowl of cereal & 80 & 1 tbsp. mayonnaise & 80 & Scrambled egg & 70 & Med banana & 60 \\
\hline & & & 90 & & & & \\
\hline Total & 460 & ------------ & 550 & ----------- & 430 & ----------- & 440 \\
\hline
\end{tabular}


Breakfast

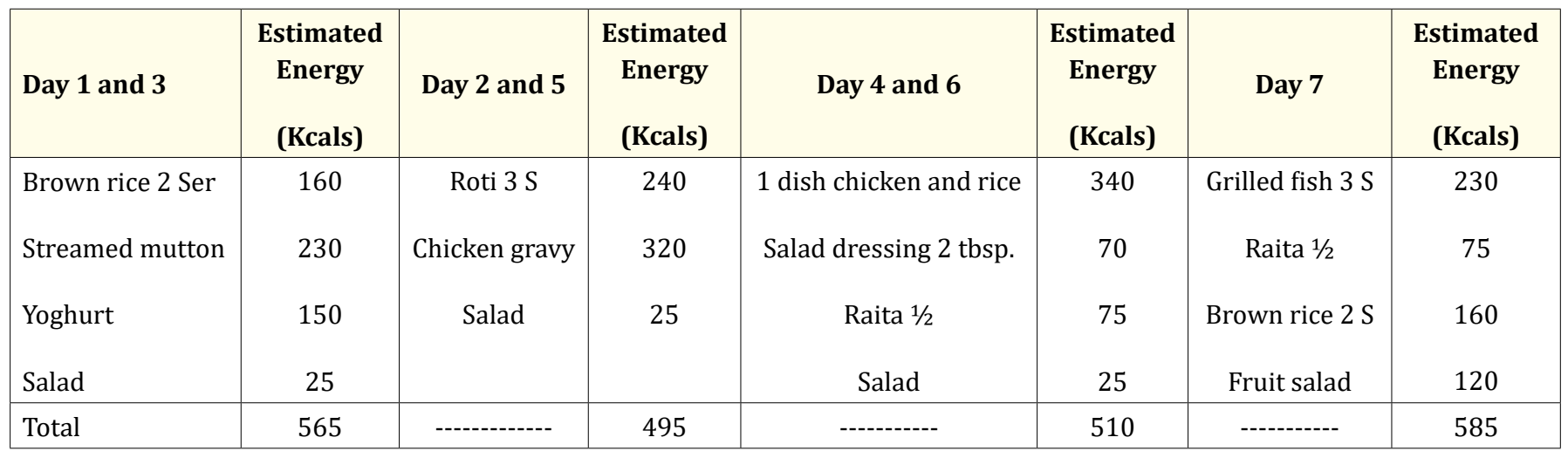

Lunch

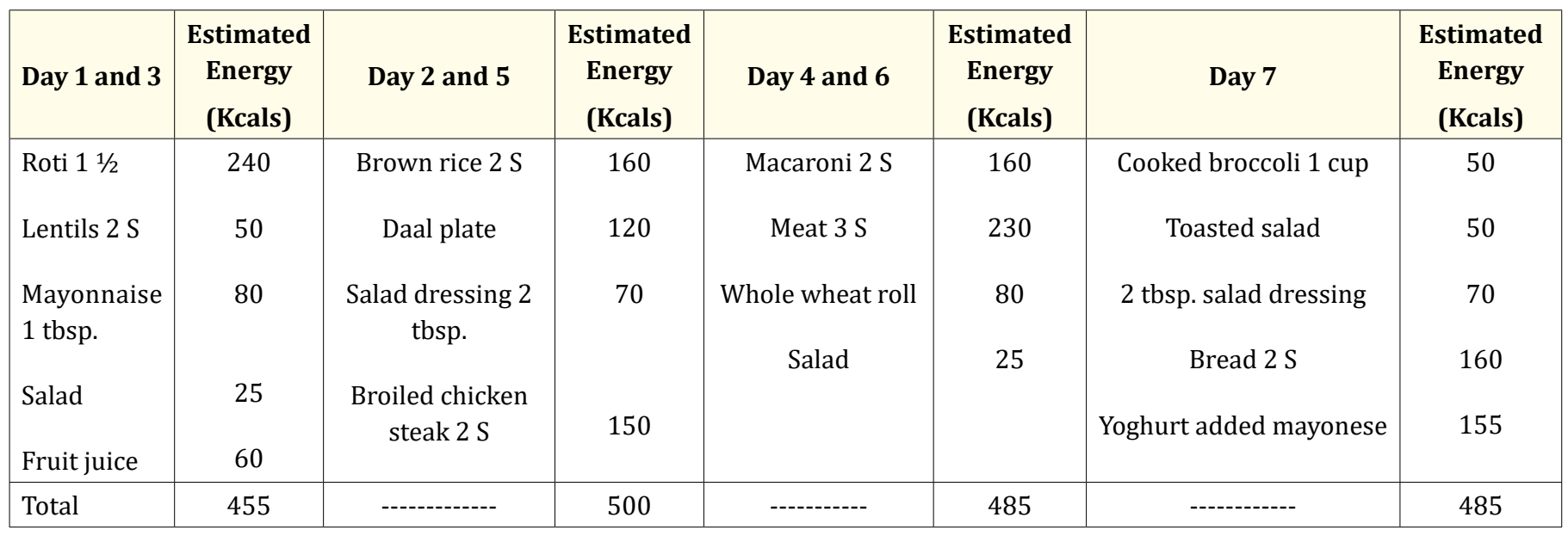

Dinner

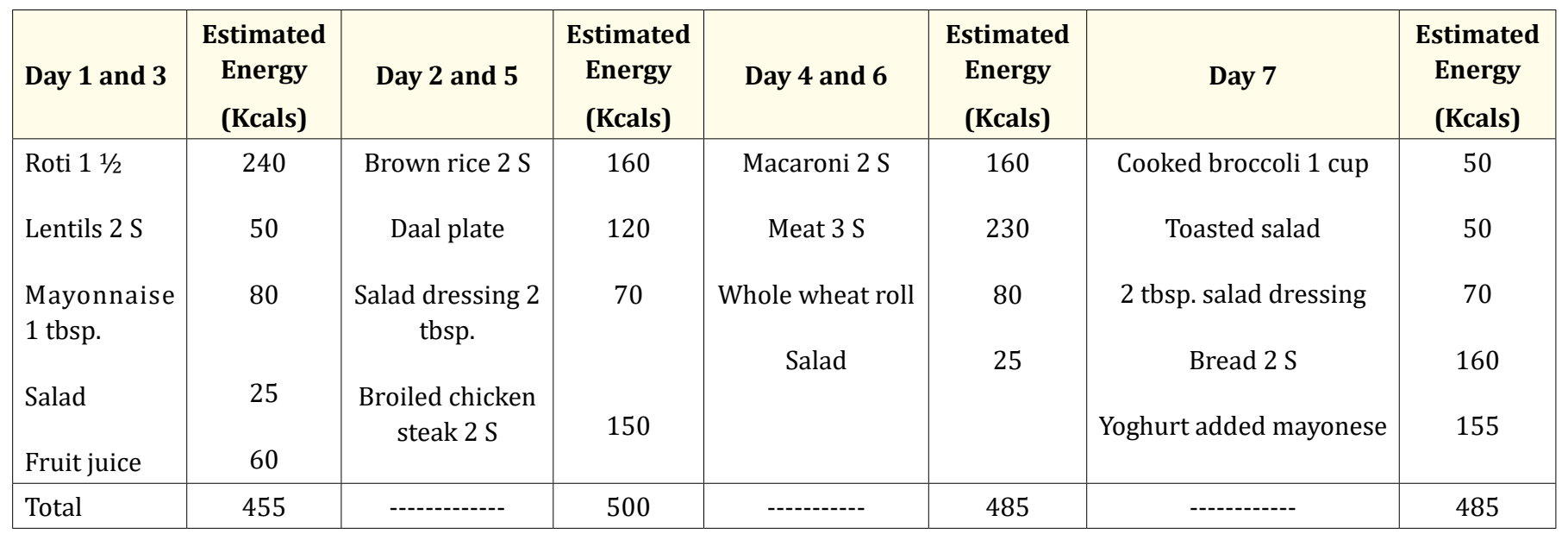


Snack times

Total Estimated Kcals of Day 1 and $3=1900$

Total Estimated Kcals of Day 2 and $5=1835$

Total Estimated Kcals of Day 4 and 6= 1949

Total Estimated Kcals of Day 7= 1940

Diet plan for cirrhosis

\begin{tabular}{|c|c|c|c|c|c|c|c|}
\hline Day 1 and 3 & $\begin{array}{c}\text { Estimated } \\
\text { Energy } \\
\text { (Kcals) }\end{array}$ & Day 2 and 5 & $\begin{array}{c}\text { Estimated } \\
\text { Energy } \\
\text { (Kcals) }\end{array}$ & Day 4 and 6 & $\begin{array}{c}\text { Estimated } \\
\text { Energy } \\
\text { (Kcals) }\end{array}$ & Day 7 & $\begin{array}{c}\text { Estimated } \\
\text { Energy } \\
\text { (Kcals) }\end{array}$ \\
\hline Bowl of cereal & 80 & Chicken sandwich & 320 & Egg & 70 & Bread 2 slices & 160 \\
\hline Far free milk & 150 & Banana shake & 210 & Bread $2 \mathrm{~S}$ & 160 & Egg & 70 \\
\hline $\begin{array}{l}\text { Fresh straw- } \\
\text { berries }\end{array}$ & 60 & & & Milk fat free & 150 & $\begin{array}{l}2 \text { tbsp. mayon- } \\
\text { naise }\end{array}$ & 180 \\
\hline Scrambled egg & 70 & & & $\begin{array}{l}\text { Bowl of fruit } \\
\text { (fresh) }\end{array}$ & 60 & Fruit juice & 60 \\
\hline Bre & 160 & & & & & & \\
\hline Total & 520 & 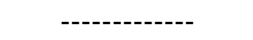 & 530 & 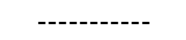 & 440 & ---.---.-- & 470 \\
\hline
\end{tabular}

\section{Breakfast}

\begin{tabular}{|l|c|c|c|c|c|c|c|}
\hline Day 1 and 3 & $\begin{array}{c}\text { Estimated } \\
\text { Energy } \\
\text { (Kcals) }\end{array}$ & Day 2 and 5 & $\begin{array}{c}\text { Estimated } \\
\text { Energy } \\
\text { (Kcals) }\end{array}$ & Day 4 and 6 & $\begin{array}{c}\text { Estimated } \\
\text { Energy }\end{array}$ & $\begin{array}{c}\text { Estimated } \\
\text { Energy } \\
\text { (Kcals) }\end{array}$ & Bay 7 \\
(Kcals)
\end{tabular}


Lunch

\begin{tabular}{|c|c|c|c|c|c|c|c|}
\hline Day 1 and 3 & $\begin{array}{c}\text { Estimated } \\
\text { Energy } \\
\text { (Kcals) }\end{array}$ & Day 2 and 5 & $\begin{array}{c}\text { Estimated } \\
\text { Energy } \\
\text { (Kcals) }\end{array}$ & Day 4 and 6 & $\begin{array}{c}\text { Estimated } \\
\text { Energy } \\
\text { (Kcals) }\end{array}$ & Day 7 & $\begin{array}{c}\text { Estimated } \\
\text { Energy } \\
\text { (Kcals) }\end{array}$ \\
\hline $\begin{array}{l}\text { Fresh broccoli } \\
\text { Cooked }\end{array}$ & 50 & Brown rice $2 \mathrm{~S}$ & 160 & $\begin{array}{c}\text { Scrambled } \\
\text { vegetable egg }\end{array}$ & 130 & Steamed brown rice $2 \mathrm{~S}$ & \\
\hline Roti $1 \frac{1}{2}$ & 240 & Pulse & 120 & Bread 2 S & 160 & Grilled fish $2 \mathrm{~S}$ & 230 \\
\hline Mashed potatoes & 80 & $\begin{array}{c}\text { Salad } 2 \text { tbsp. fat } \\
\text { free salad dressing }\end{array}$ & 70 & Raita & 150 & Raita & 150 \\
\hline 1 cup & & Yoghurt fat free & 150 & Fruit juice & 60 & Salad & 25 \\
\hline Salad & 25 & & & & & & \\
\hline $\begin{array}{l}\text { Unsweetened } \\
\text { fruit juice }\end{array}$ & 60 & & & & & & \\
\hline Total & 455 & --.---.-- & 500 & --------- & 500 & ---------- & 565 \\
\hline
\end{tabular}

Dinner

\begin{tabular}{|l|c|c|c|c|c|c|}
\hline Days & Snack Time 1 & $\begin{array}{c}\text { Estimated } \\
\text { Energy } \\
\text { (Kcals) }\end{array}$ & Snack Time 2 & $\begin{array}{c}\text { Estimated } \\
\text { Energy } \\
\text { (Kcals) }\end{array}$ & Snack Time 3 & $\begin{array}{c}\text { Estimated } \\
\text { Energy } \\
\text { (Kcals) }\end{array}$ \\
\hline Day 1 and 3 & $\begin{array}{c}\text { Tea with saltine } \\
\text { cracker }\end{array}$ & 80 & Cheese & 140 & Milk & 150 \\
\hline Day 2 and 5 & Fruit cocktail & 120 & Flavored yoghurt & 150 & Med Apple & 60 \\
\hline Day 4 and 6 & Almond 1S & 164 & Ice cream 1/2 cup & 140 & Low fat milk & 150 \\
\hline Day 7 & Fruit salad & 120 & Med apple & 70 & custard & 200 \\
\hline
\end{tabular}

\section{Snack times}

Total Estimated Kcals of Day 1 and $3=1845$

Total Estimated Kcals of Day 2 and $5=1900$

Total Estimated Kcals of Day 4 and $6=1954$

Total Estimated Kcals of Day $7=1965$.

Diet plan for COPD

\begin{tabular}{|c|c|c|c|c|c|c|c|}
\hline Day 1 and 3 & $\begin{array}{c}\text { Estimated } \\
\text { Energy } \\
\text { (Kcals) }\end{array}$ & Day 2 and 5 & $\begin{array}{c}\text { Estimated } \\
\text { Energy } \\
\text { (Kcals) }\end{array}$ & Day 4 and 6 & $\begin{array}{c}\text { Estimated } \\
\text { Energy } \\
\text { (Kcals) }\end{array}$ & Day 7 & $\begin{array}{c}\text { Estimated } \\
\text { Energy } \\
\text { (Kcals) }\end{array}$ \\
\hline Toast 2S & 160 & Boiled egg & 70 & 2 slice bread & 160 & Cheese sandwich & 235 \\
\hline 1 glass milk shake & 210 & Chicken sandwich & 310 & Yoghurt 1S & 150 & Med. apple & 60 \\
\hline Egg & 70 & Milk & 150 & Omelet & 165 & I cup cereal & 80 \\
\hline & & & & 2 tbs oil & & Milk $1 / 2 \mathrm{~S}$ & 75 \\
\hline Total & 440 & ------------- & 530 & ---------- & 475 & ----------- & 450 \\
\hline
\end{tabular}


Breakfast

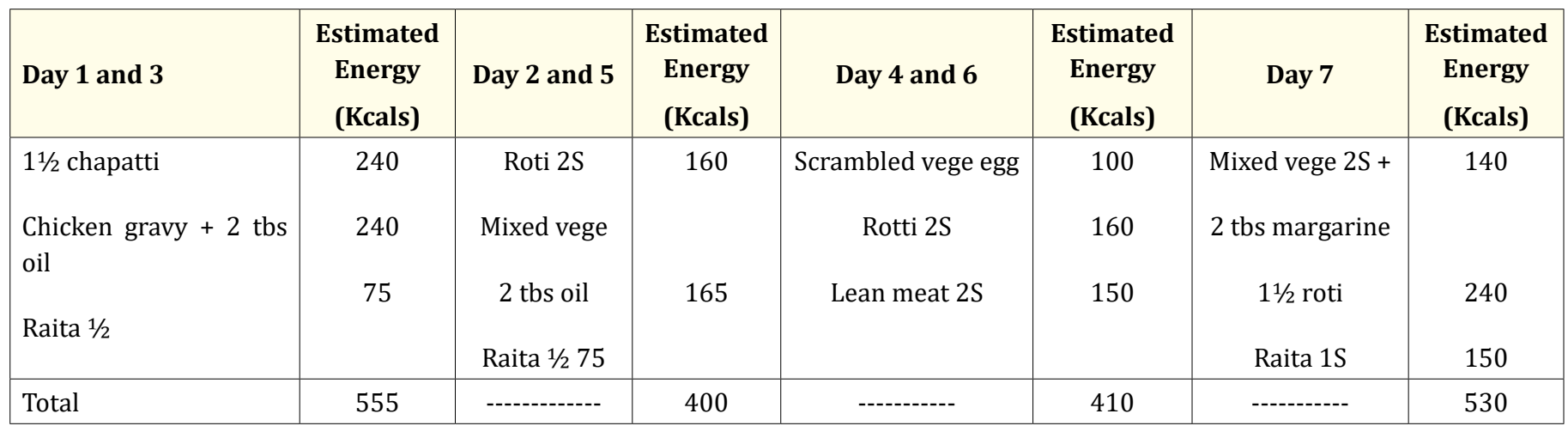

Lunch

\begin{tabular}{|c|c|c|c|c|c|c|c|}
\hline Day 1 and 3 & $\begin{array}{c}\text { Estimated } \\
\text { Energy } \\
\text { (Kcals) }\end{array}$ & Day 2 and 5 & $\begin{array}{c}\text { Estimated } \\
\text { Energy } \\
\text { (Kcals) }\end{array}$ & Day 4 and 6 & $\begin{array}{c}\text { Estimated } \\
\text { Energy } \\
\text { (Kcals) }\end{array}$ & Day 7 & $\begin{array}{c}\text { Estimated } \\
\text { Energy } \\
\text { (Kcals) }\end{array}$ \\
\hline Total & 465 & ------------ & 480 & --------- & 550 & ----------- & 540 \\
\hline
\end{tabular}

Dinner

\begin{tabular}{|l|c|c|c|c|c|c|}
\hline Days & Snack Time 1 & $\begin{array}{c}\text { Estimated } \\
\text { Energy } \\
\text { (Kcals) }\end{array}$ & Snack Time 2 & $\begin{array}{c}\text { Estimated } \\
\text { Energy } \\
\text { (Kcals) }\end{array}$ & Snack Time 3 & $\begin{array}{c}\text { Estimated } \\
\text { Energy } \\
\text { (Kcals) }\end{array}$ \\
\hline Day 1 and 3 & Fruit cocktail & 120 & Banana med. 2S & 120 & Milk & 150 \\
\hline Day 2 and 5 & Almond 1S & 164 & Flavored yoghurt 1/2 & 75 & Banana shake & 210 \\
\hline Day 4 and 6 & Berries & 60 & Fruit juice & 60 & Pudding & 147 \\
\hline Day 7 & Tea + saltine cracker & 80 & Med. apple & 60 & Milk & 150 \\
\hline
\end{tabular}

Snack times

Total Estimated Kcals of Day 1 and 3= 1850

Total Estimated Kcals of Day 2 and $5=1860$

Total Estimated Kcals of Day 4 and $6=1702$

Total Estimated Kcals of Day 7= 1810. 
Diet plan for obesity

\begin{tabular}{|c|c|c|c|c|c|c|c|}
\hline Day 1 and 3 & $\begin{array}{c}\text { Estimated } \\
\text { Energy } \\
\text { (Kcals) }\end{array}$ & Day 2 and 5 & $\begin{array}{c}\text { Estimated } \\
\text { Energy } \\
\text { (Kcals) }\end{array}$ & Day 4 and 6 & $\begin{array}{c}\text { Estimated } \\
\text { Energy } \\
\text { (Kcals) }\end{array}$ & Day 7 & $\begin{array}{c}\text { Estimated } \\
\text { Energy } \\
\text { (Kcals) }\end{array}$ \\
\hline Skimmed milk $1 \mathrm{~S}$ & 91 & Skimmed milk $1 \mathrm{~S}$ & 91 & 1 whole wheat muffin & 80 & Skimmed milk & 91 \\
\hline Orange med & 60 & Banana med & 60 & Skimmed milk & 91 & Scrambled egg & 75 \\
\hline 1 cup cereal & 80 & 1 cup bran cereal & 80 & $1 / 2$ cup blue berries & 60 & 2 slice bread & 160 \\
\hline Total & 231 & 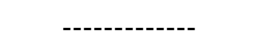 & 231 & ---------- & 231 & ----------- & 326 \\
\hline
\end{tabular}

Breakfast

\begin{tabular}{|c|c|c|c|c|c|c|c|}
\hline Day 1 and 3 & $\begin{array}{c}\text { Estimated } \\
\text { Energy } \\
\text { (Kcals) }\end{array}$ & Day 2 and 5 & $\begin{array}{c}\text { Estimated } \\
\text { Energy } \\
\text { (Kcals) }\end{array}$ & Day 4 and 6 & $\begin{array}{c}\text { Estimated } \\
\text { Energy } \\
\text { (Kcals) }\end{array}$ & Day 7 & $\begin{array}{c}\text { Estimated } \\
\text { Energy } \\
\text { (Kcals) }\end{array}$ \\
\hline $\begin{array}{l}\text { Roti } 1 \mathrm{~S} \\
\text { Chicken vege mix } \\
\text { Skimmed yoghurt } 1 / 2 \mathrm{~S}\end{array}$ & $\begin{array}{l}80 \\
175 \\
68\end{array}$ & $\begin{array}{l}\text { Steamed chicken } 1 \mathrm{~S} \\
\text { Raita } 1 / 2 \\
\text { Roti } 2 \mathrm{~S}\end{array}$ & $\begin{array}{l}75 \\
75 \\
160\end{array}$ & $\begin{array}{l}\text { Chicken rice } \\
\text { one dish } \\
1 / 2 \text { Raita }\end{array}$ & $\begin{array}{l}310 \\
75\end{array}$ & $\begin{array}{l}\text { Vegetable rice } \\
1 / 2 \text { Raita }\end{array}$ & $\begin{array}{l}210 \\
75\end{array}$ \\
\hline Total & 224 & ----------- & 310 & --------- & 385 & --------- & 285 \\
\hline
\end{tabular}

Lunch

\begin{tabular}{|c|c|c|c|c|c|c|c|}
\hline Day 1 and 3 & $\begin{array}{c}\text { Estimated } \\
\text { Energy } \\
\text { (Kcals) }\end{array}$ & Day 2 and 5 & $\begin{array}{c}\text { Estimated } \\
\text { Energy } \\
\text { (Kcals) }\end{array}$ & Day 4 and 6 & $\begin{array}{c}\text { Estimated } \\
\text { Energy } \\
\text { (Kcals) }\end{array}$ & Day 7 & $\begin{array}{c}\text { Estimated } \\
\text { Energy } \\
\text { (Kcals) }\end{array}$ \\
\hline Roti $2 S$ & 160 & Brown rice $2 \mathrm{~S}$ & 160 & Grilled fish 2S & 150 & Chicken +vege 1tbsp oil & 160 \\
\hline $\begin{array}{l}\text { Mix Vege cooked } \\
\text { with 2tbsp oil }\end{array}$ & 140 & $\begin{array}{l}\text { Daal } \\
\text { Salad }\end{array}$ & $\begin{array}{l}120 \\
20\end{array}$ & $\begin{array}{l}\text { Roti } 1 \mathrm{~S} \\
\text { Raita } 1 / 2\end{array}$ & $\begin{array}{l}150 \\
75 \\
\end{array}$ & Roti 1S & 150 \\
\hline Total & 300 & ------------ & 300 & ---------- & 375 & ---------- & 310 \\
\hline
\end{tabular}

Dinner

\begin{tabular}{|l|c|c|c|c|c|c|}
\hline Days & Snack Time 1 & $\begin{array}{c}\text { Estimated } \\
\text { Energy } \\
\text { (Kcals) }\end{array}$ & Snack Time 2 & $\begin{array}{c}\text { Estimated } \\
\text { Energy } \\
\text { (Kcals) }\end{array}$ & Snack Time 3 & $\begin{array}{c}\text { Estimated } \\
\text { Energy } \\
\text { (Kcals) }\end{array}$ \\
\hline Day 1 and 3 & Tea with saltine cracker & 80 & Grapes & 80 & Skimmed milk & 91 \\
\hline Day 2 and 5 & 1 kiwi & 60 & Biscuit with tea & 80 & Apple & 60 \\
\hline Day 4 and 6 & Melon 1 cup & 60 & Lemon juice & 60 & Skimmed milk & 91 \\
\hline Day 7 & Banana & 60 & Peach med & 60 & Ice cream & 102 \\
\hline
\end{tabular}


Snack times

Total Estimated Kcals of Day 1 and 3= 1143

Total Estimated Kcals of Day 2 and $5=1202$

Total Estimated Kcals of Day 4 and 6=1041

Total Estimated Kcals of Day $7=1006$.

\section{Conclusion}

In this study, I have been very good experience for me and I have learned how to eat good and healthy food and how to eat this food with diet plan and if we have some disease so we should follow this diet plan it gives you benefits and its good role in your health.

Volume 3 Issue 12 December 2019

(C) All rights are reserved by Murium Sultan., et al. 